Saudi Journal of Medical and Pharmaceutical Sciences

Abbreviated Key Title: Saudi J Med Pharm Sci

ISSN 2413-4929 (Print) |ISSN 2413-4910 (Online)

Scholars Middle East Publishers, Dubai, United Arab Emirates

Journal homepage: https://saudijournals.com

Case Report

\title{
Giant Cystic Lymphangioma in Children: About Two Cases
}

Ahmed Ould Mohamed ${ }^{1},{ }^{*}$, Bencheikh Razika ${ }^{2},{ }^{3}$, Gliti Mohamed $\mathrm{Ali}^{1},{ }^{3}$, Benbouzid Mohamed Anas ${ }^{2},{ }^{3}$, Leila Essakalli Houssyni ${ }^{2},^{3}$

${ }^{1}$ Resident Physician in Otorhinolaryngology, Department of Otorhinolaryngology, Head and Neck Surgery, Ibn Sina University Hospital, Rabat, Morocco

${ }^{2}$ Professor of Otorhinolaryngology, Department of Otorhinolaryngology, Head and Neck Surgery, Ibn Sina University Hospital, Rabat, Morocco

${ }^{3}$ Faculty of Medicine and Pharmacy of Rabat, Mohammed V University, Rabat, Morocco

DOI: $10.36348 /$ sjmps.2021.v07i01.014

| Received: 04.01.2021 | Accepted: 18.01.2021 | Published: 29.01.2021

*Corresponding author: Ahmed Ould Mohamed

\section{Abstract}

Objective: describe the therapeutic modalities and surgical management of children with giant cystic lymphangioma. Materials and methods: These are two cases of a child with congenital giant cystic lymphangioma, respectively 14 months and 2 years old, treated in the otorhinolaryngology and neck and neck surgery department at the university hospital in rabat. Conclusion: Cervicofacial cystic lymphangiomas are benign tumor malformations of congenital origin. Their pathogenesis remains debated, the centrifugal theory being the most accepted. Their suspected positive diagnosis on clinical examination and medical imaging can only be confirmed by intervention and histological examination of the surgical specimen. Thanks to recent clinical-radiological staging, their management may involve surgery or sclerotherapy, depending on the indication. Without being a neonatal emergency, in general, the intervention must be early and complete, as the tumor is progressive, to ensure complete healing and the absence of recurrence.

Keywords: Cystic lymphangiomas, cervical mass, congenital, child.

Copyright ( ) 2021 The Author(s): This is an open-access article distributed under the terms of the Creative Commons Attribution 4.0 International License (CC BY-NC 4.0) which permits unrestricted use, distribution, and reproduction in any medium for non-commercial use provided the original author and source are credited.

\section{INTRODUCTION}

Cystic lymphangiomas are rare benign dysembryoplasias of the lymph node system, responsible for a tumor syndrome by angiolymphatic proliferation [1]. They are included, in the new classification of the ISSVA of vascular malformations, in the context of low flow lymphatic malformations [2]. This condition, first reported in 1828 by Redenbacher 1 , is better known since the reference work by Sabin in 1909 and 1912.

Their anatomical location is almost exclusively cervicofacial [3], and their clinical revelation is generally very early [1].The severity of these tumor formations in children is due, on the one hand, to their evolutionary potential liable to compress and invade the upper aerodigestive tract, and on the other hand, by the classic difficulty of their resection, which then becomes partial.

The clinic radiological staging of Serres [5]. has made it possible to predict the rate of complications, and recently to carry out management algorithms [3,5].whose main modalities are therapeutic abstention, surgical excision, and sclerotherapy, except in cases of emergency.

\section{Case Report}

Case 1

A 14-month-old infant who presents since birth bilateral sublingual tumefaction and oral floor tumefaction, requiring hospitalization in neonatal intensive care for two days, with the placement of a gastric tube for 5 months. Then he was bottle-feeding.

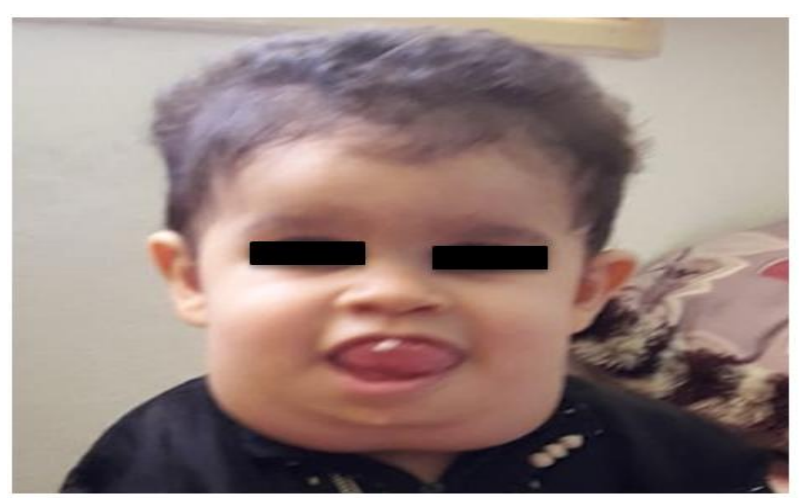

Fig-1: Photographic image of the infant showing edema at the submandibular and upper cervical level

Citation: Ahmed Ould Mohamed et al (2021). Giant Cystic Lymphangioma in Children: About Two Cases. Saudi J Med Pharm Sci, 7(1): 71-76. 71 
The evolution of the mass was progressive without signs of compression (without dyspnea, dysphagia, or dysphonia).

The clinical examination does not find any limitation of the mouth opening or protrusion of the tongue, with the presence of a tumefaction in the floor of the mouth.
The cervicothoracic scanner found a mass of fluid density occupying the sublingual space and the well-limited right submandibular fossa with a regular contour containing finely enhanced septum after injection of the contrast product evoking cystic lymphangioma.
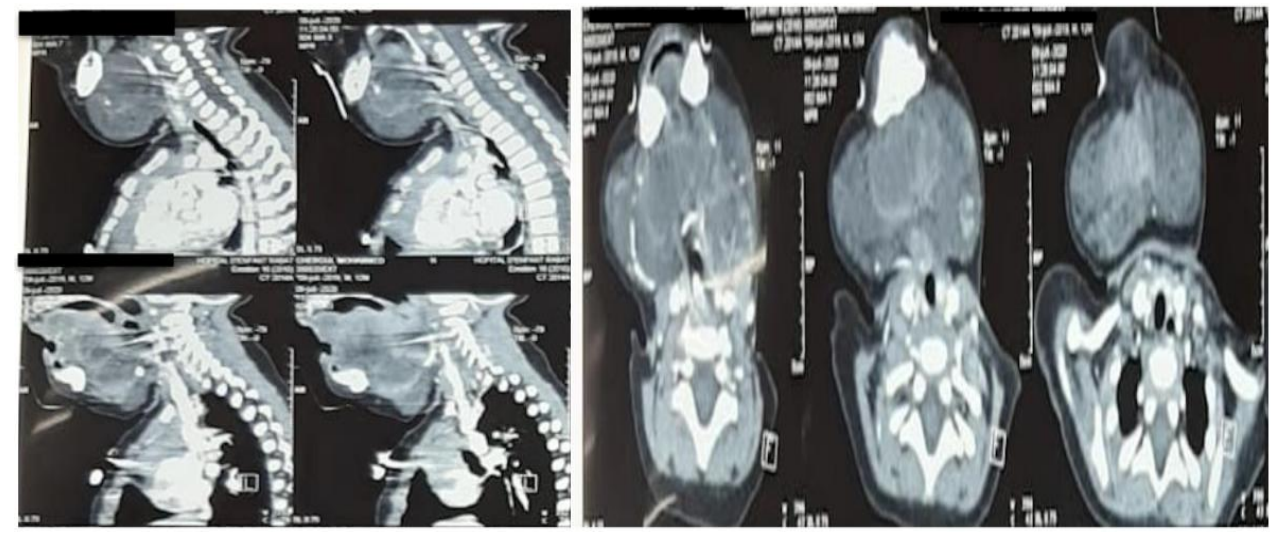

Fig-2: Image of the cervico-thoracic CT scan in axial, sagital and coronal section, with injection of the contrast product, shows a formation of fluid density, multi-partitioned

The therapeutic management consisted of complete surgical excision. The immediate post-op is complicated by a reaction macro glossy which gradually regresses after administration of oral corticosteroid treatment.

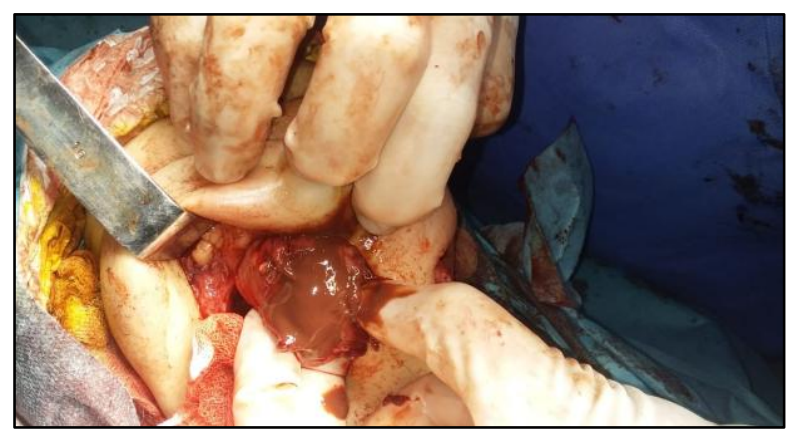

Fig-3: Intraoperative image showing the viscous brown contents of the sublingual cystic formation

Case 2:

A 2 years old child, hospitalized for left lateral cervical swelling. The onset of cervical swelling goes back to birth, gradually increasing in size, recently associated with a dry cough. No dyspnea, no dysphagia, no dysphonia, or cervicobrachial irritation. On examination, a right cervical mass was found, measuring $3 * 4 \mathrm{~cm}$. These masses had a soft consistency, a lilac color of the skin opposite, and they were mobile concerning the two planes. The examination found bronchopulmonary congestion, crackles, and apical dullness on the left side.

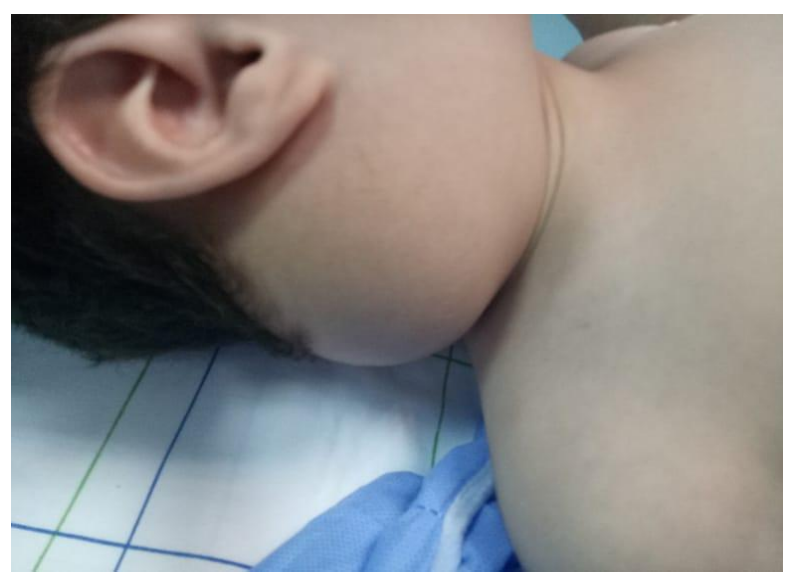

Fig-4: Photographic image showing a right latero cervical mass, renitante; healthy skin opposite, without turgor of the jugular veins, of the posterior cervical triangle

The cervical CT with an injection of the product of contrast found an enormous mass of fluid density pregnant all the right parotid region and cervical. 


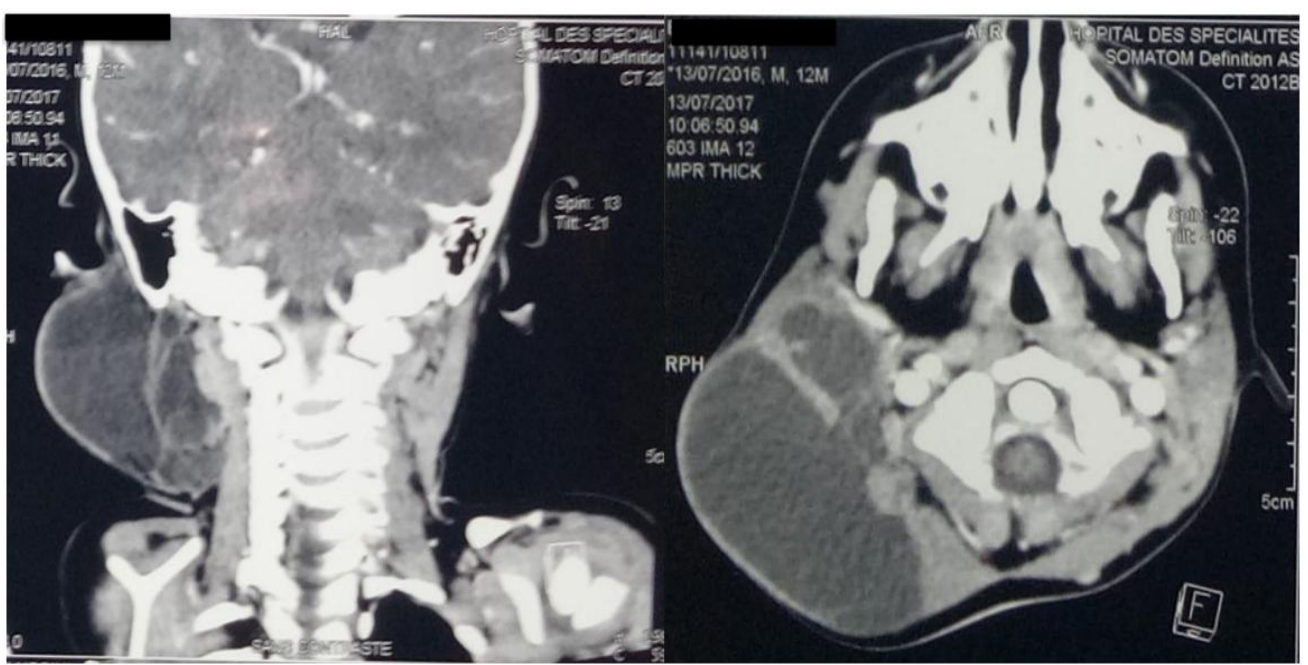

Fig-5: Image of cervical CT scan with injection of contrast product showing enormous mass of fluid density pregnant all the right parotid region and cervical

The therapeutic management consisted of surgical resection, with good postoperative improvement.

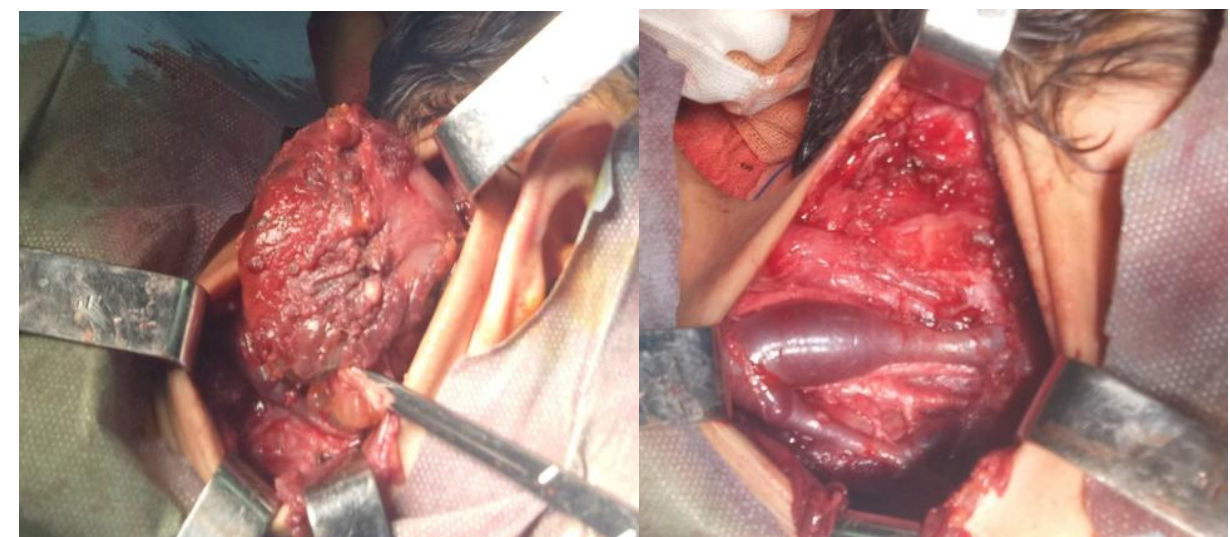

Fig-6: Intraoperative image showing the extent of the mass with significant internal jugular venous dilation

\section{DISCUSSION}

Cystic lymphangiomas are rare benign tumors that can occur in any region of the body, but it is the cervicofacial locations that predominate with $75 \%$ of cases for Gugliantini et al., [4], 65\% of cases for Bill and Summer [5], and $62.2 \%$ of cases for Kennedy et al., [6].

They represent between 2.6 and $5 \%$ of congenital benign cervical masses [7].

Their frequency is 17 cases in 20 years reported by Ozen et al., [8]. Triglia et al., reported three cases per year [9] and Tekou et al., 1.8 cases in one year [10]. In the neck, cystic lymphangioma is classically located in the posteroinferior triangle [11].

The locations of cystic lymphangiomas mainly concern the submandibular region. For François et al., [2], the anterior locations (50\%) are as frequent as the posterior locations. Supra-hyoid cystic lymphangiomas fall within the scope of diffuse lymphangiomatosis, the deformities of which are sometimes less visible [43]. In these forms, it is necessary to look for an invasion of the parotid, the cheek, the tonsillar space, the parapharyngeal space, the tongue, or the larynx. Isolated jugal and parotid localizations are rare and pose the problem of recurrences and aesthetic sequelae postoperatively.

Although of dysembryoplastic origin, the cyst is present at birth in only 50 to $60 \%$ of cases, it is expressed in $90 \%$ of cases before the age of two [2].

The average occurrence age reported by Uba and Children is five months [12], it is significantly lower than that found by Raji et al., (five years) [11]. Some lymphangiomas do not appear until late in adulthood as evidenced by studies by Raji et al., [13] and Diop et al., [14]. The sex ratio varies from series to series. a female predominance is found in the series by François et al., [2] and by Raji et al., [11], but they contrast with those of Triglia et al., [9] who report an equal distribution between the two sexes and those of Ameh and Nmadu [15] who report male predominance. 
The diagnosis of cystic lymphangioma, in our context, is most often made from clinical and intraoperative data. Some exams are useful for looking for a deep extension. Standard cervicothoracic radiography is not particularly important. She is looking for a tracheal deviation or mediastinal extension. Cervical ultrasound provides more information [19]. It specifies the size, the number of cystic pockets, and their multi partitioned character. It allows, by the analysis of echogenicity, to assess the content of different pockets which are most often hypoechoic, fluid without Doppler flow, and rarely echogenic in the event of inflammatory flare and intracystic hemorrhage. The multi-compartmentalized aspect is very suggestive of the diagnosis. However, the tumor extension is sometimes difficult to determine on ultrasound due to the often large tumor size. This information is best appreciated by $\mathrm{CT}$ and magnetic resonance imaging [19].

The scanner looks for deep sub mylohyoid, mediastinal, laryngeal, parapharyngeal, and cranial basal extensions. It makes it possible to find a multicloisonné mass, of fluid density, not capturing the contrast product except at the level of the walls [19]. MRI seems to give more specific images with a T2 hyper signal reproducing the different partitions. Above all, it allows a precise analysis of the extensions to the mediastinum, the parotid region, and the parapharyngeal regions as well as the relationship of the cyst to the vasculonervous elements of the neck [20].

The treatment of cervicofacial cystic lymphangiomas is classically surgical $[2,8,9,18]$. The variety of cervical and facial incisions, which the head and neck surgeon are accustomed to, allow them to expose and remove all pockets of the cyst. Let us simply recall the L-shaped incisions of Paul-André, Sebileau-Carrega, and Redon which allow a broader approach and the control of the vascular-nervous element elements of the neck and the face. There is still a debate; this is the best age for this surgery. Some authors [21] estimate that it is necessary to wait until the age of six to nine months for general anesthesia to be less risky. Some belief, on the other hand, that early surgery is preferable, before the inflammatory or bleeding flare-ups make dissection difficult. Indeed, surgical excision can be easy at an early stage when it is mono or pauci cystic form limited to the neck. It is much more difficult in cavernous, microcystic, or extensive lymphangiomas. The surgical technique must therefore obey the rules of lymph-ganglionic neck surgery, which is always long and meticulous.

It must be part of a conservative or modified radical cystocellulo ganglion recess [9]. This is because cystic lymphangioma has an intimate relationship with the cervical venous system, the thoracic duct, or the large lymphatic vein, and there is no real plane of cleavage. Often thinned and atrophic muscles lose their role as beacons in cervical surgery. Performing atraumatic conservative surgery, therefore, requires a trained surgeon, mastering not only neck surgery but also that of neighboring regions such as the parotid, the pterygomaxillary fossa, the larynx, the floor of the mouth, the base of the tongue, and the upper mediastinum. The surgical procedure must be, as much as possible, complete to avoid recurrence. However, the notion of complete excision should not put the patient at risk of postoperative functional and aesthetic effects for a condition that remains benign. These risks are more frequent for parotid localizations, as the dissection of the facial nerve is made difficult by the tumor which encompasses it. Lymphangiomas located in the cheek and lip also pose the problem of an appropriate approach and that of repair when the mucosa is invaded. These are generally rather vascularized microcystic lymphangiomas of the hemolymphangioma type and rarely surgical excision is complete. Therefore, some authors [6] suggest the opening or marsupialization of cysts that could not be resected to cause their atrophy.

Complications from surgery are all the more important to consider as cystic lymphangioma is a benign tumor. The center- or postoperative mortality varies from 0 to $7 \%$ depending on the series $[5,6,14$, 22]. It is all the more important as the number of reoperations increases and it is a younger child. This calls for caution in extended forms, in particular at the base of the tongue, at the vallecula, or at the laryngeal rim where the impossibility of intubation makes it necessary to perform a tracheotomy. Local complications are responsible for long consequences. These are lymphorrhea and lymphedema. Other local complications, such as loose sutures, hematoma, or superinfection, can occur [2].

Neurological sequelae vary depending on the series. Ozen et al., [8] report $12 \%$ in 17 cases. Raji et al., [11] report on 15 cases, two cases of paralysis of the chin branch of the facial, and one case of spinal paralysis. Ameh and Nmadu [15] report on 41 cases, two cases of facial paralysis, and one case of recurrent nerve palsy.

The main problem with cystic lymphangioma is postoperative recurrence. It is seen especially after incomplete resection with $36 \%$ of cases against $6 \%$ in the case of complete resection [2, 5, 7, 22]. This multiplies the surgical procedures in even more difficult conditions.

Faced with these surgical difficulties, Mikhail et al., [23] used a sclerosing product (OK-432) in two patients after incomplete excisional surgery with good results. Some authors have used sclerosing products first-line in the treatment of cystic lymphangiomas. 
Martinot et al., [24] reported 16 good results in 20 patients treated by percutaneous injection of Ethibloc®. Fermand and kuttenberger [25] report three good results in four patients treated by intratumoral injection of triamcinolone. Oxford et al., [26] reported seven complete cures in 16 patients treated by intratumoral injection of bleomycin. However, these products are only effective for other authors [9, 24, 27] in macro cystic lymphangiomas.

The CO2 laser is used by centers that have it in laryngeal, vallecular, and basilinguales lesions [3]. A radiotherapy is no longer an option because it has not been proven to be effective and also carries a carcinogenic risk in children [9].

Apart from inflammatory outbreaks, where antibiotic corticosteroid therapy is prescribed, the rapid increase in volume responsible for respiratory distress sometimes requires punctures to relieve the tension of the cysts. If necessary, a life-saving tracheostomy is indicated [28]. These are all waiting solutions for curative surgery.

\section{CONCLUSION}

Cervicofacial cystic lymphangiomas are benign tumor malformations of congenital origin. Their pathogenesis remains debated, the centrifugal theory being the most accepted.

Their suspected positive diagnosis on clinical examination and medical imaging can only be confirmed by intervention and histological examination of the surgical specimen. Thanks to recent clinicalradiological staging, their management may involve surgery or sclerotherapy, depending on the indication.

Without being a neonatal emergency, in general, the intervention must be early and complete, as the tumor is progressive, to ensure complete healing and the absence of recurrence.

\section{REFERENCES}

1. Sabin, F. R. (1912). In: The lymphatic system in human embryos with a consideration of the morphology of the system as a whole. Philadelphie, J. P. Lipspincott company. 2:70915.

2. François, M., Le Guillou, C., Depondt, J., Aboucaya, J. P., \& Contencin, P. (1986). Les lymphangiomes cervico-faciaux chez l'enfant. In Annales d'oto-laryngologie et de chirurgie cervico-faciale (Vol. 103, No. 2, pp. 113-117).

3. Hartl, D. M., Roger, G., Denoyelle, F., Nicollas, R., Triglia, J. M., \& Garabedian, E. N. (2000). Extensive lymphangioma presenting with upper airway obstruction. Archives of OtolaryngologyHead \& Neck Surgery, 126(11), 1378-1382.
4. Gugliantini, P., Fariello, G., \& D'Onofrio, M. (1975). Cystic hygroma of the neck exploration with iodinated contrast media. In Annales de radiologie (Vol. 18, No. 4, p. 453).

5. Bill, A. H. (1965). A unified concept of lymphangioma and cystic hygroma. Surg Gynecol Obstet, 120, 79-86.

6. Kennedy, T. L., Whitaker, M., Pellitteri, P., \& Wood, W. E. (2001). Cystic hygroma/lymphangioma: a rational approach to management. The laryngoscope, 111(11), 19291937.

7. Bock, M. E., Smith, J. M., Parey, S. E., \& Mobley, D. L. (1987). Lymphangiome. An otolaryngologic. Int Pediatr Otolaryngol, 14, 133144.

8. Ozen, I. O., Moralioglu, S., Karabulut, R., Demirogullari, B., Sonmez, K., Turkyilmaz, Z., ... \& Kale, N. (2005). Surgical treatment of cervicofacial cystic hygromas in children. ORL, 67(6), 331-334.

9. Raji, A., Essaadi, M., Touhami, M., Chekkoury, I., \& Benchakroun, Y. (2000). Les lymphangiomes kystiques cervico-faciaux de l'enfant: A propos de 15 cas. Maghreb médical, (346), 133-136.

10. Tekou, H., Maboodou, A., Feteke, L., Foly, A., Senah, K. C., \& Atanley, R. (1998). Les lymphangiomes kystiques du cou de l'enfant à Lomé. Tunis. Med., 76, 24-27.

11. Raji, A., Essaadi, M., Touhami, M., CHEKKOURY, I., \& Benchakroun, Y. (2000). Les lymphangiomes kystiques cervico-faciaux de l'enfant: A propos de 15 cas. Maghreb médical, (346), 133-136.

12. Uba, A. F., \& Chirdan, L. B. (2006). Management of cystic lymphangioma in children: experience in Jos, Nigeria. Pediatric surgery international, 22(4), 353-356.

13. Raji, A., Essaadi, M., Mahtar, M., Roubal, M., \& Chekkoury, I. A. (2002). Les lymphangiomes cervico-faciaux de l'adulte (à propos de 10 cas). Revue de laryngologie, d'otologie et de rhinologie (1919), 123(1), 27-32.

14. Diop, E. M., Diouf, R., \& Diop, L. (1984). La lymphangiome kystique: un problème pratique de l'oncologie cervicale. A propos de 4 observations. In Annales d'oto-laryngologie et de chirurgie cervico-faciale (Vol. 101, No. 2, pp. 109-113).

15. Ameh, E. A., \& Nmadu, P. T. (2001). Cervical cystic hygroma: pre-, intra-, and post-operative morbidity and mortality in Zaria, Nigeria. Pediatric surgery international, 17(5-6), 342-343.

16. Fisher, R., Partington, A., \& Dykes, E. (1996). Cystic hygroma: comparison between prenatal and postnatal diagnosis. Journal of pediatric surgery, 31(4), 473-476.

17. Ricciardelli, E. J., \& Richardson, M. A. (1991). Cervicofacial cystic hygroma: patterns of 
recurrence and management of the difficult case. Archives of Otolaryngology-Head \& Neck Surgery, 117(5), 546-553.

18. Pia, F., Aluffi, P., \& Olina, M. (1999). Cystic lymphangioma in the head and neck region. Acta otorhinolaryngologica Italica: organo ufficiale della Societa italiana di otorinolaringologia e chirurgia cervico-facciale, 19(2), 87-90.

19. Anne, F., Hurtier, O., Garcia, J. F., Filippini, J. F., \& Piriou, A. (1992, January). Cystic lymphangioma of the neck in adults. Contribution of ultrasonography and computed tomography. Apropos of a case. In Annales de Radiologie (Vol. 35, No. 4, pp. 212-216).

20. Fung, K., Poenaru, D., Soboleski, D. A. A., \& Kamal, I. M. (1998). Impact of magnetic resonance imaging on the surgical management of cystic hygromas. Journal of pediatric surgery, 33(6), 839-841.

21. Grabb, W. C., Dingman, R. O., Oneal, R. M., \& Dempsey, P. D. (1980). Facial hamartomas in children: neurofibroma, lymphangioma, and hemangioma. Plastic and reconstructive surgery, 66(4), 509-527.

22. Hancock, B. J., St-Vil, D., Luks, F. I., Di Lorenzo, M., \& Blanchard, H. (1992). Complications of lymphangiomas in children. Journal of pediatric surgery, 27(2), 220-226.

23. Mikhail, M., Kennedy, R., Cramer, B., \& Smith,
T. (1995). Sclerosing of recurrent lymphangioma using OK-432. Journal of pediatric surgery, 30(8), 1159-1160.

24. Martinot, V., Descamps, S., Février, P., Patenotre, P., Brevière, J. M., Piette, F., \& Pellerin, P. (1997). Évaluation du traitement des lymphangiomes kystiques par injection percutanée d'Éthibloc ${ }^{\circledR}$ chez 20 patients. Archives de pédiatrie, 4(1), 8-14.

25. Farmand, M., \& Kuttenberger, J. J. (1996). A new therapeutic concept for the treatment of cystic hygroma. Oral Surgery, Oral Medicine, Oral Pathology, Oral Radiology, and Endodontology, 81(4), 389-395.

26. Orford, J., Barker, A., Thonell, S., King, P., \& Murphy, J. (1995). Bleomycin therapy for cystic hygroma. Journal of pediatric surgery, 30(9), 1282-1287.

27. Giguère, C. M., Bauman, N. M., Sato, Y., Burke, D. K., Greinwald, J. H., Pransky, S., ... \& Smith, R. J. (2002). Treatment of lymphangiomas with OK-432 (Picibanil) sclerotherapy: a prospective multi-institutional trial. Archives of Otolaryngology-Head \& Neck Surgery, 128(10), 1137-1144.

28. Orvidas, L. J., \& Kasperbauer, J. L. (2000). Pediatric lymphangiomas of the head and neck. Annals of Otology, Rhinology \& Laryngology, 109(4), 411-421. 